\title{
NEW PROPERTIES OF THE JUNG-KIM-SRIVASTAVA INTEGRAL OPERATORS
}

\author{
B. A. FRASIN
}

Abstract. The object of the present paper is to prove new subordinations results of analytic functions defined by two integral operators $P^{\alpha}$ and $Q_{\beta}^{\alpha}$. Several corollaries and consequences of the main results are also considered.

\section{Introduction and definitions}

Let $\mathscr{A}$ denote the class of functions of the form :

$$
f(z)=z+\sum_{n=2}^{\infty} a_{n} z^{n}
$$

which are analytic in the open unit disc $\mathscr{U}=\{z:|z|<1\}$. For the functions $f$ and $g$ in $\mathscr{A}$, we say that $f$ is subordinate to $g$ in $\mathscr{U}$, and write $f<g$, if there exists a Schwarz function $w(z)$, which (by definition) is analytic in $\mathscr{U}$ with $|w(z)|<1$ and $w(0)=0$ such that $f(z)=g(w(z))$ in $\mathscr{U}$.

Recently, Jung et al. [4] have introduced the following one-parameter families of integral operators:

$$
\begin{aligned}
& P^{\alpha} f=P^{\alpha} f(z)=\frac{2^{\alpha}}{z \Gamma(\alpha)} \int_{0}^{z}\left(\log \frac{z}{t}\right)^{\alpha-1} f(t) d t \quad(\alpha>0) \\
& Q_{\beta}^{\alpha} f=Q_{\beta}^{\alpha} f(z)=\left(\begin{array}{c}
\alpha+\beta \\
\beta
\end{array}\right) \frac{\alpha}{z^{\beta}} \int_{0}^{z}\left(1-\frac{t}{z}\right)^{\alpha-1} t^{\beta-1} f(t) d t \quad(\alpha>0, \beta>-1)
\end{aligned}
$$

and

$$
J_{\alpha} f=J_{\alpha} f(z)=\frac{\alpha+1}{z^{\alpha}} \int_{0}^{z} t^{\alpha-1} f(t) d t \quad(\alpha>-1)
$$

For $\alpha \in \mathbb{N}=\{1,2,3, \ldots\}$, the operators $P^{\alpha}, Q_{1}^{\alpha}$ and $J_{\alpha}$ were considered by Bernardi ([2], [3]). Further, for real number $\alpha>-1$, the operator $J_{\alpha}$ was used by Owa and Srivastava [6], and by Srivastava and Owa ([7], [8]).

2000 Mathematics Subject Classification. 30C45.

Key words and phrases. Analytic functions, linear operator, differential subordination. 
For $f(z) \in \mathscr{A}$ Jung et al.[4] have shown that

$$
\begin{aligned}
& P^{\alpha} f(z)=z+\sum_{n=2}^{\infty}\left(\frac{2}{n+1}\right)^{\alpha} a_{n} z^{n} \quad(\alpha>0), \\
& Q_{\beta}^{\alpha} f(z)=z+\frac{\Gamma(\alpha+\beta+1)}{\Gamma(\beta+1)} \sum_{n=2}^{\infty} \frac{\Gamma(\beta+n)}{\Gamma(\alpha+\beta+n)} a_{n} z^{n} \quad(\alpha>0, \beta>-1)
\end{aligned}
$$

and

$$
J_{\alpha} f(z)=z+\sum_{n=2}^{\infty}\left(\frac{\alpha+1}{\alpha+n}\right) a_{n} z^{n} \quad(\alpha>-1) .
$$

By virtue of (1.5) and (1.7), we see that

$$
J_{\alpha} f(z)=Q_{\alpha}^{1} f(z) \quad(\alpha>-1) .
$$

In this paper is we derive new subordinations results of analytic functions defined by the above linear operators $P^{\alpha}$ and $Q_{\beta}^{\alpha}$.

In order to prove our main results, we recall the following lemmas:

Lemma 1.1 ([5]). If $p(z)=1+p_{1}(z)+p_{2}(z)+\ldots$ is analytic in $\mathscr{U}$ and $h(z)$ is a convex function in $\mathscr{U}$ with $h(0)=1$ and $\gamma$ is a complex constant such that $\operatorname{Re}(\gamma)>0$, then

$$
p(z)+\frac{z p^{\prime}(z)}{\gamma} \prec h(z)
$$

implies

$$
p(z) \prec \gamma z^{-\gamma} \int_{o}^{z} t^{\gamma-1} h(t) d t=q(z) \prec h(z)
$$

and $q(z)$ is the best dominant.

Lemma 1.2 ([1]). For $a, b, c$ real numbers other than $0,-1,-2, \ldots$, and $c>b>0$, we have

$$
\begin{aligned}
& \int_{0}^{1} t^{b-1}(1-t)^{c-b-1}(1-t z)^{-a} d t=\frac{\Gamma(b) \Gamma(c-b)}{\Gamma(c)} F(a, b ; c ; z) \\
& F(a, b ; c ; z)=(1-z)^{-a} F\left(a, c-b ; c ; \frac{z}{z-1}\right) \\
& F(1,1 ; 2 ; z)=-z^{-1} \ln (1-z) \\
& c(c-1)(z-1) F(a, b ; c-1 ; z)+c[c-1-(2 c-a-b-1) z] F(a, b ; c ; z) \\
& \quad+(c-a)(c-b) z F(a, b ; c+1 ; z)=0 .
\end{aligned}
$$

From the identities (1.13) and (1.14), we easily prove the following

Lemma 1.3. For any real number $\zeta \neq 0$, we have

$$
F\left(1,1 ; 2 ; \frac{\zeta z}{\zeta z+1}\right)=\frac{(1+\zeta z) \ln (1+\zeta z)}{\zeta z}
$$




$$
\begin{aligned}
& F\left(1,1 ; 3 ; \frac{\zeta z}{\zeta z+1}\right)=\frac{2(1+\zeta z)}{\zeta z}\left[1-\frac{\ln (1+\zeta z)}{\zeta z}\right] \\
& F\left(1,1 ; 4 ; \frac{\zeta z}{\zeta z+1}\right)=\frac{3(1+\zeta z)}{2(\zeta z)^{3}}[2 \ln (1+\zeta z)-\zeta z(2-\zeta z)] \\
& F\left(1,1 ; 5 ; \frac{\zeta z}{\zeta z+1}\right)=\frac{2(1+\zeta z)}{(\zeta z)^{3}}\left[\frac{2(\zeta z)^{2}-3 \zeta z+6}{3}-\frac{2 \ln (1+\zeta z)}{\zeta z}\right] .
\end{aligned}
$$

\section{Main results}

Theorem 2.1. Let $\lambda>0, \alpha>2$ and suppose that

$$
\frac{P^{\alpha-1} f(z)}{P^{\alpha} f(z)}\left(1+\lambda\left(\frac{P^{\alpha-2} f(z)}{P^{\alpha-1} f(z)}-\frac{P^{\alpha-1} f(z)}{P^{\alpha} f(z)}\right)\right)<\frac{1+A z}{1+B z}
$$

then we have

$$
\frac{P^{\alpha-1} f(z)}{P^{\alpha} f(z)}<q(z)<\frac{1+A z}{1+B z} \quad(z \in \mathscr{U})
$$

where

$$
q(z)=(1+B z)^{-1}\left[F\left(1,1 ; 1+\frac{2}{\lambda} ; \frac{B z}{B z+1}\right)+\frac{2 A z}{2+\lambda} F\left(1,1 ; 2+\frac{2}{\lambda} ; \frac{B z}{B z+1}\right)\right]
$$

and $q(z)$ is the best dominant. Furthermore,

$$
\operatorname{Re}\left\{\frac{P^{\alpha-1} f(z)}{P^{\alpha} f(z)}\right\}>\rho
$$

where

$$
\rho=(1-B)^{-1}\left[F\left(1,1 ; 1+\frac{2}{\lambda} ; \frac{B}{B-1}\right)-\frac{2 A}{2+\lambda} F\left(1,1 ; 2+\frac{2}{\lambda} ; \frac{B}{B-1}\right)\right]
$$

Proof. Define the function $p(z)$ by

$$
p(z):=\frac{P^{\alpha-1} f(z)}{P^{\alpha} f(z)} .
$$

Then $p(z)=1+b_{1} z+b_{2} z+\cdots$ is analytic in $\mathscr{U}$ with $p(0)=1$. A computation shows that

$$
\frac{z p^{\prime}(z)}{p(z)}=\left(\frac{z\left(P^{\alpha-1} f(z)\right)^{\prime}}{P^{\alpha-1} f(z)}-\frac{z\left(P^{\alpha} f(z)\right)^{\prime}}{P^{\alpha} f(z)}\right) .
$$

By making use of the familiar identity

$$
z\left(P^{\alpha} f(z)\right)^{\prime}=2 P^{\alpha-1} f(z)-P^{\alpha} f(z) \quad(\alpha>1)
$$

in (2.5) , we get

$$
\frac{P^{\alpha-2} f(z)}{P^{\alpha-1} f(z)}-\frac{P^{\alpha-1} f(z)}{P^{\alpha} f(z)}=\frac{z p^{\prime}(z)}{2 p(z)}
$$


By using (2.4) and (2.7), we obtain

$$
\frac{P^{\alpha-1} f(z)}{P^{\alpha} f(z)}\left(1+\lambda\left(\frac{P^{\alpha-2} f(z)}{P^{\alpha-1} f(z)}-\frac{P^{\alpha-1} f(z)}{P^{\alpha} f(z)}\right)\right)=p(z)+\frac{\lambda}{2} z p^{\prime}(z) .
$$

Thus, by using Lemma 1.1 for $\gamma=\frac{2}{\lambda}$, we have

$$
\frac{P^{\alpha-1} f(z)}{P^{\alpha} f(z)} \prec\left(\frac{2}{\lambda}\right) z^{-\left(\frac{2}{\lambda}\right)} \int_{0}^{z} \frac{t^{\frac{2}{\lambda}-1}(1+A t) d t}{1+B t}=q(z) .
$$

Now using the identities (1.11) and (1.12), we can rewritten the function $q(z)$ as

$$
\begin{aligned}
q(z) & =\left(\frac{2}{\lambda}\right) \int_{0}^{1} \frac{s^{\frac{2}{\lambda}-1}(1+A s z)}{1+B s z} d s \\
& =\left(\frac{2}{\lambda}\right) \int_{0}^{1} s^{\frac{2}{\lambda}-1}(1+B s z)^{-1} d s+A\left(\frac{2}{\lambda}\right) z \int_{0}^{1} s^{\frac{2}{\lambda}}(1+B s z)^{-1} d s \\
& =(1+B z)^{-1}\left[F\left(1,1 ; 1+\frac{2}{\lambda} ; \frac{B z}{B z+1}\right)+\frac{2 A z}{2+\lambda} F\left(1,1 ; 2+\frac{2}{\lambda} ; \frac{B z}{B z+1}\right)\right] .
\end{aligned}
$$

This completes the proof of (2.1).

Next to prove (2.3), it suffices to show that

$$
\inf _{|z|<1}\{q(z)\}=q(-1)
$$

Since for $-1 \leq B<A \leq 1,(1+A z) /(1+B z)$ is convex (univalent) in $\mathscr{U}$, we have for $|z| \leq r<1$,

$$
\operatorname{Re}\left(\frac{1+A z}{1+B z}\right) \geq \frac{1-A r}{1-B r}
$$

Upon setting

$$
g(s, z)=\frac{1+A s z}{1+B s z}, \quad(0 \leq s \leq 1, z \in \mathscr{U})
$$

and

$$
d v(s)=s^{\left(\frac{2}{\lambda}-1\right)}\left(\frac{2}{\lambda}\right) d s
$$

which is a positive measure on $[0,1]$, we get

$$
q(z)=\int_{0}^{1} g(s, z) d v(s)
$$

so that

$$
\operatorname{Re}\{q(z)\} \geq \int_{0}^{1}\left(\frac{1-A s r}{1-B s r}\right) d v(s)=q(-r), \quad(|z| \leq r<1) .
$$

Letting $r \rightarrow 1^{-}$in the above inequality, we obtain the assertion (2.8). Hence the Theorem.

Letting $\lambda=2$ in Theorem 2.1 and using the identities (1.15) and (1.16), we have 
Corollary 2.2. Let $\alpha>2$ and suppose that

$$
\frac{P^{\alpha-1} f(z)}{P^{\alpha} f(z)}\left(1+2\left(\frac{P^{\alpha-2} f(z)}{P^{\alpha-1} f(z)}-\frac{P^{\alpha-1} f(z)}{P^{\alpha} f(z)}\right)\right) \prec \frac{1+A z}{1+B z}
$$

then we have

$$
\frac{P^{\alpha-1} f(z)}{P^{\alpha} f(z)}<q(z)<\frac{1+A z}{1+B z} \quad(z \in \mathscr{U})
$$

where

$$
q(z)= \begin{cases}\frac{A}{B}+\left(1-\frac{A}{B}\right) \frac{\ln (1+B z)}{B z}, & B \neq 0 \\ 1+\frac{A}{2} z, & B=0\end{cases}
$$

and $q(z)$ is the best dominant. Furthermore,

$$
\operatorname{Re}\left\{\frac{P^{\alpha-1} f(z)}{P^{\alpha} f(z)}\right\}>\rho,
$$

where

$$
\rho=\left\{\begin{array}{ll}
\frac{A}{B}-\left(1-\frac{A}{B}\right) \frac{\ln (1-B)}{B}, & B \neq 0 \\
1-\frac{A}{2}, & B=0 .
\end{array} .\right.
$$

Letting $\lambda=1$ in Theorem 2.1 and using the identities (1.16) and (1.17), we have

Corollary 2.3. Let $\alpha>2$ and suppose that

$$
\frac{P^{\alpha-1} f(z)}{P^{\alpha} f(z)}\left(1+\frac{P^{\alpha-2} f(z)}{P^{\alpha-1} f(z)}-\frac{P^{\alpha-1} f(z)}{P^{\alpha} f(z)}\right)<\frac{1+A z}{1+B z}
$$

then we have

$$
\frac{P^{\alpha-1} f(z)}{P^{\alpha} f(z)} \prec q(z) \prec \frac{1+A z}{1+B z} \quad(z \in \mathscr{U})
$$

where

$$
q(z)= \begin{cases}\frac{A}{B}-\frac{2}{B^{2}}\left(1-\frac{A}{B}\right)\left[\frac{\ln (1+B z)-B z}{z^{2}}\right], & B \neq 0 \\ 1+\frac{2 A}{3} z, & B=0\end{cases}
$$

and $q(z)$ is the best dominant. Furthermore,

$$
\operatorname{Re}\left\{\frac{P^{\alpha-1} f(z)}{P^{\alpha} f(z)}\right\}>\rho,
$$

where

$$
\rho=\left\{\begin{array}{ll}
\frac{A}{B}-\frac{2}{B^{2}}\left(1-\frac{A}{B}\right)[\ln (1-B)+B], & B \neq 0 \\
1-\frac{2 A}{3}, & B=0
\end{array} .\right.
$$

Letting $\lambda=2 / 3$ in Theorem 2.1 and using the identities (1.17) and (1.18), we have 
Corollary 2.4. Let $\alpha>2$ and suppose that

$$
\frac{P^{\alpha-1} f(z)}{P^{\alpha} f(z)}\left(1+\frac{2}{3}\left(\frac{P^{\alpha-2} f(z)}{P^{\alpha-1} f(z)}-\frac{P^{\alpha-1} f(z)}{P^{\alpha} f(z)}\right)\right) \prec \frac{1+A z}{1+B z}
$$

then we have

$$
\frac{P^{\alpha-1} f(z)}{P^{\alpha} f(z)}<q(z)<\frac{1+A z}{1+B z} \quad(z \in \mathscr{U})
$$

where

$$
q(z)= \begin{cases}\frac{A}{B}+\frac{3}{(B z)^{3}}\left(1-\frac{A}{B}\right)\left[\ln (1+B z)-B z+\frac{(B z)^{2}}{2}\right], & B \neq 0 \\ 1+\frac{3 A}{4} z, & B=0\end{cases}
$$

and $q(z)$ is the best dominant. Furthermore,

$$
\operatorname{Re}\left\{\frac{P^{\alpha-1} f(z)}{P^{\alpha} f(z)}\right\}>\rho
$$

where

$$
\rho= \begin{cases}\frac{A}{B}-\frac{3}{B^{3}}\left(1-\frac{A}{B}\right)\left[\ln (1-B)+B-\frac{B^{2}}{2}\right], & B \neq 0 \\ 1-\frac{3 A}{4}, & B=0\end{cases}
$$

Letting $B \neq 0$ in Corollaries 2.2, 2.3 and 2.4, respectively, we obtain the following :

Corollary 2.5. Let $\alpha>2$, then we have the following:

$$
\begin{aligned}
& \text { If } \frac{P^{\alpha-1} f(z)}{P^{\alpha} f(z)}\left(1+2\left(\frac{P^{\alpha-2} f(z)}{P^{\alpha-1} f(z)}-\frac{P^{\alpha-1} f(z)}{P^{\alpha} f(z)}\right)\right) \prec \frac{1+\frac{B \ln (1-B)}{B+\ln (1-B)} z}{1+B z} \\
& \Rightarrow \operatorname{Re}\left\{\frac{P^{\alpha-1} f(z)}{P^{\alpha} f(z)}\right\}>0 \text { in } \mathscr{U} .
\end{aligned}
$$

(ii) If $\frac{P^{\alpha-1} f(z)}{P^{\alpha} f(z)}\left(1+\frac{P^{\alpha-2} f(z)}{P^{\alpha-1} f(z)}-\frac{P^{\alpha-1} f(z)}{P^{\alpha} f(z)}\right) \prec \frac{1+\frac{2 B[B+\ln (1-B)]}{2(B+\ln (1-B))+B^{2}} z}{1+B z}$ $\Rightarrow \operatorname{Re}\left\{\frac{P^{\alpha-1} f(z)}{P^{\alpha} f(z)}\right\}>0$ in $\mathscr{U}$.

(iii) If $\frac{P^{\alpha-1} f(z)}{P^{\alpha} f(z)}\left(1+\frac{2}{3}\left(\frac{P^{\alpha-2} f(z)}{P^{\alpha-1} f(z)}-\frac{P^{\alpha-1} f(z)}{P^{\alpha} f(z)}\right)\right) \prec \frac{1+\frac{3 B\left[\ln (1-B)+B-\left(B^{2} / 2\right)\right]}{B^{3}+3\left[\ln (1-B)+B-\left(B^{2} / 2\right)\right]} z}{1+B z}$ $\Rightarrow \operatorname{Re}\left\{\frac{P^{\alpha-1} f(z)}{P^{\alpha} f(z)}\right\}>0$ in $\mathscr{U}$.

Letting $B=-1$ in Corollary 2.5, we have

Corollary 2.6. Let $\alpha>2$, then we have the following: 
(i) If $\operatorname{Re}\left\{\frac{P^{\alpha-1} f(z)}{P^{\alpha} f(z)}\left(1+2\left(\frac{P^{\alpha-2} f(z)}{P^{\alpha-1} f(z)}-\frac{P^{\alpha-1} f(z)}{P^{\alpha} f(z)}\right)\right)\right\}>\frac{2 \ln 2-1}{2 \ln 2-2} \approx-0.61$

$\Rightarrow \operatorname{Re}\left\{\frac{P^{\alpha-1} f(z)}{P^{\alpha} f(z)}\right\}>0$ in $\mathscr{U}$.

(ii) If $\operatorname{Re}\left\{\frac{P^{\alpha-1} f(z)}{P^{\alpha} f(z)}\left(1+\frac{P^{\alpha-2} f(z)}{P^{\alpha-1} f(z)}-\frac{P^{\alpha-1} f(z)}{P^{\alpha} f(z)}\right)\right\}>\frac{4 \ln 2-3}{4 \ln 2-2} \approx-0.29$

$\Rightarrow \operatorname{Re}\left\{\frac{P^{\alpha-1} f(z)}{P^{\alpha} f(z)}\right\}>0$ in $\mathscr{U}$

(iii) If $\operatorname{Re}\left\{\frac{P^{\alpha-1} f(z)}{P^{\alpha} f(z)}\left(1+\frac{2}{3}\left(\frac{P^{\alpha-2} f(z)}{P^{\alpha-1} f(z)}-\frac{P^{\alpha-1} f(z)}{P^{\alpha} f(z)}\right)\right)\right\}>\frac{12 \ln 2-19}{12 \ln 2-20} \approx 0.91$

$\Rightarrow \operatorname{Re}\left\{\frac{P^{\alpha-1} f(z)}{P^{\alpha} f(z)}\right\}>0$ in $\mathscr{U}$

Letting $A=1-2 \delta, 0 \leq \delta<1$ and $B=-1$ in Corollaries2.2, 2.3 and 2.4, respectively, we have

Corollary 2.7. Let $\alpha>2$, then we have the following:

(i) If $\operatorname{Re}\left\{\frac{P^{\alpha-1} f(z)}{P^{\alpha} f(z)}\left(1+2\left(\frac{P^{\alpha-2} f(z)}{P^{\alpha-1} f(z)}-\frac{P^{\alpha-1} f(z)}{P^{\alpha} f(z)}\right)\right)\right\}>\delta$

$\Rightarrow \operatorname{Re}\left\{\frac{P^{\alpha-1} f(z)}{P^{\alpha} f(z)}\right\}>(2 \delta-1)+2(1-\delta) \ln 2$.

(ii) If $\operatorname{Re}\left\{\frac{P^{\alpha-1} f(z)}{P^{\alpha} f(z)}\left(1+\frac{P^{\alpha-2} f(z)}{P^{\alpha-1} f(z)}-\frac{P^{\alpha-1} f(z)}{P^{\alpha} f(z)}\right)\right\}>\delta$

$\Rightarrow \operatorname{Re}\left\{\frac{P^{\alpha-1} f(z)}{P^{\alpha} f(z)}\right\}>(2 \delta-1)-4(1-\delta)(\ln 2-1)$

(iii) If $\operatorname{Re}\left\{\frac{P^{\alpha-1} f(z)}{P^{\alpha} f(z)}\left(1+\frac{2}{3}\left(\frac{P^{\alpha-2} f(z)}{P^{\alpha-1} f(z)}-\frac{P^{\alpha-1} f(z)}{P^{\alpha} f(z)}\right)\right)\right\}>\delta$

$\Rightarrow \operatorname{Re}\left\{\frac{P^{\alpha-1} f(z)}{P^{\alpha} f(z)}\right\}>(2 \delta-1)+3(1-\delta)(2 \ln 2-3)$.

Theorem 2.8. Let $\alpha>2, \beta>-1, \lambda>0$ and suppose that

$$
\frac{Q_{\beta}^{\alpha-1} f(z)}{Q_{\beta}^{\alpha} f(z)}\left(1+\lambda\left(\frac{Q_{\beta}^{\alpha-2} f(z)}{Q_{\beta}^{\alpha-1} f(z)}-\frac{\alpha+\beta}{\alpha+\beta-1} \frac{Q_{\beta}^{\alpha-1} f(z)}{Q_{\beta}^{\alpha} f(z)}+\frac{1}{\alpha+\beta-1}\right)\right)<\frac{1+A z}{1+B z}
$$

then we have

$$
\frac{Q_{\beta}^{\alpha-1} f(z)}{Q_{\beta}^{\alpha} f(z)} \prec q(z) \prec \frac{1+A z}{1+B z} \quad(z \in \mathscr{U})
$$

where

$$
q(z)=(1+B z)^{-1}\left[F\left(1,1 ; 1+\frac{\alpha+\beta-1}{\lambda} ; \frac{B z}{B z+1}\right)+\frac{(\alpha+\beta-1) A z}{\alpha+\beta-1+\lambda} F\left(1,1 ; 2+\frac{\alpha+\beta-1}{\lambda} ; \frac{B z}{B z+1}\right)\right]
$$


and $q(z)$ is the best dominant. Furthermore,

$$
\operatorname{Re}\left\{\frac{Q_{\beta}^{\alpha-1} f(z)}{Q_{\beta}^{\alpha} f(z)}\right\}>\rho,
$$

where

$$
\rho=(1-B)^{-1}\left[F\left(1,1 ; 1+\frac{\alpha+\beta-1}{\lambda} ; \frac{B}{B-1}\right)-\frac{(\alpha+\beta-1) A}{\alpha+\beta-1+\lambda} F\left(1,1 ; 2+\frac{\alpha+\beta-1}{\lambda} ; \frac{B}{B-1}\right)\right] .
$$

Proof. Define the function $p(z)$ by

$$
p(z):=\frac{Q_{\beta}^{\alpha-1} f(z)}{Q_{\beta}^{\alpha} f(z)} .
$$

Then $p(z)=1+b_{1} z+b_{2} z+\cdots$ is analytic in $\mathscr{U}$ with $p(0)=1$. Also, by a simple computation and by making use of the familiar identity

$$
z\left(Q_{\beta}^{\alpha} f(z)\right)^{\prime}=(\alpha+\beta) Q_{\beta}^{\alpha-1} f(z)-(\alpha+\beta-1) Q_{\beta}^{\alpha} f(z) \quad(\alpha>1, \beta>-1)
$$

we find from (2.25) that

$$
\lambda\left(\frac{Q_{\beta}^{\alpha-2} f(z)}{Q_{\beta}^{\alpha-1} f(z)}-\frac{\alpha+\beta}{\alpha+\beta-1} \frac{Q_{\beta}^{\alpha-1} f(z)}{Q_{\beta}^{\alpha} f(z)}+\frac{1}{\alpha+\beta-1}\right)=\left(\frac{\lambda}{\alpha+\beta-1}\right) \frac{z p^{\prime}(z)}{p(z)}
$$

by using (2.25) and (2.27), we get

$$
\begin{aligned}
& \frac{Q_{\beta}^{\alpha-1} f(z)}{Q_{\beta}^{\alpha} f(z)}\left(1+\lambda\left(\frac{Q_{\beta}^{\alpha-2} f(z)}{Q_{\beta}^{\alpha-1} f(z)}-\frac{\alpha+\beta}{\alpha+\beta-1} \frac{Q_{\beta}^{\alpha-1} f(z)}{Q_{\beta}^{\alpha} f(z)}+\frac{1}{\alpha+\beta-1}\right)\right) \\
& \quad=p(z)+\left(\frac{\lambda}{\alpha+\beta-1}\right) z p^{\prime}(z) .
\end{aligned}
$$

Using Lemma 1.1 for $\gamma=\frac{\alpha+\beta-1}{\lambda}$, the estimates (2.23) and (2.24) can be proved on the same lines as that of (2.1) and (2.3). Hence the theorem.

Letting $\lambda=1, \alpha=2-\beta$ in Theorem 2.8 and using the identities (1.15) and (1.16), we have Corollary 2.9. Let $-1<\beta<0$ and suppose that

$$
\frac{Q_{\beta}^{1-\beta} f(z)}{Q_{\beta}^{2-\beta} f(z)}\left(2+\frac{Q_{\beta}^{-\beta} f(z)}{Q_{\beta}^{1-\beta} f(z)}-2 \frac{Q_{\beta}^{1-\beta} f(z)}{Q_{\beta}^{2-\beta} f(z)}\right) \prec \frac{1+A z}{1+B z}
$$

then we have

$$
\frac{Q_{\beta}^{1-\beta} f(z)}{Q_{\beta}^{2-\beta} f(z)} \prec q(z) \prec \frac{1+A z}{1+B z} \quad(z \in \mathscr{U})
$$


where $q(z)$ as given in (2.12) and $q(z)$ is the best dominant. Furthermore,

$$
\operatorname{Re}\left\{\frac{Q_{\beta}^{1-\beta} f(z)}{Q_{\beta}^{2-\beta} f(z)}\right\}>\rho,
$$

where $\rho$ as given in (2.13).

Letting $\lambda=1, \alpha=3-\beta$ in Theorem 2.8 and using the identities (1.16) and (1.17), we have

Corollary 2.10. Let $-1<\beta<1$ and suppose that

$$
\frac{Q_{\beta}^{2-\beta} f(z)}{Q_{\beta}^{3-\beta} f(z)}\left(\frac{3}{2}+\frac{Q_{\beta}^{1-\beta} f(z)}{Q_{\beta}^{2-\beta} f(z)}-\frac{3}{2} \frac{Q_{\beta}^{2-\beta} f(z)}{Q_{\beta}^{3-\beta} f(z)}\right) \prec \frac{1+A z}{1+B z}
$$

then we have

$$
\frac{Q_{\beta}^{2-\beta} f(z)}{Q_{\beta}^{3-\beta} f(z)}<q(z) \prec \frac{1+A z}{1+B z} \quad(z \in \mathscr{U})
$$

where $q(z)$ as given in (2.16) and $q(z)$ is the best dominant. Furthermore,

$$
\operatorname{Re}\left\{\frac{Q_{\beta}^{2-\beta} f(z)}{Q_{\beta}^{3-\beta} f(z)}\right\}>\rho,
$$

where $\rho$ as given in (2.17).

Letting $\lambda=1, \alpha=4-\beta$ in Theorem 2.8 and using the identities (1.17) and (1.18), we have Corollary 2.11. Let $-1<\beta<2$ and suppose that

$$
\frac{Q_{\beta}^{3-\beta} f(z)}{Q_{\beta}^{4-\beta} f(z)}\left(\frac{4}{3}+\frac{Q_{\beta}^{2-\beta} f(z)}{Q_{\beta}^{3-\beta} f(z)}-\frac{4}{3} \frac{Q_{\beta}^{3-\beta} f(z)}{Q_{\beta}^{4-\beta} f(z)}\right) \prec \frac{1+A z}{1+B z}
$$

then we have

$$
\frac{Q_{\beta}^{3-\beta} f(z)}{Q_{\beta}^{4-\beta} f(z)}<q(z) \prec \frac{1+A z}{1+B z} \quad(z \in \mathscr{U})
$$

where $q(z)$ as given in (2.20) and $q(z)$ is the best dominant. Furthermore,

$$
\operatorname{Re}\left\{\frac{Q_{\beta}^{3-\beta} f(z)}{Q_{\beta}^{4-\beta} f(z)}\right\}>\rho,
$$

where $\rho$ as given in (2.21). 
Letting $B \neq 0$ in Corollaries 2.9, 2.10 and 2.11, respectively, we obtain the following :

Corollary 2.12. (i) $L e t-1<\beta<0$. If $\frac{Q_{\beta}^{1-\beta} f(z)}{Q_{\beta}^{2-\beta} f(z)}\left(2+\frac{Q_{\beta}^{-\beta} f(z)}{Q_{\beta}^{1-\beta} f(z)}-2 \frac{Q_{\beta}^{1-\beta} f(z)}{Q_{\beta}^{2-\beta} f(z)}\right)$

$$
\prec \frac{1+\frac{B \ln (1-B)}{B+\ln (1-B)} z}{1+B z} \Rightarrow \operatorname{Re}\left\{\frac{Q_{\beta}^{1-\beta} f(z)}{Q_{\beta}^{2-\beta} f(z)}\right\}>0 \text { in } \mathscr{U}
$$

(ii) Let $-1<\beta<1$. If $\frac{Q_{\beta}^{2-\beta} f(z)}{Q_{\beta}^{3-\beta} f(z)}\left(\frac{3}{2}+\frac{Q_{\beta}^{1-\beta} f(z)}{Q_{\beta}^{2-\beta} f(z)}-\frac{3}{2} \frac{Q_{\beta}^{2-\beta} f(z)}{Q_{\beta}^{3-\beta} f(z)}\right)$

$$
\prec \frac{1+\frac{2 B[B+\ln (1-B)]}{2(B+\ln (1-B))+B^{2}} z}{1+B z} \Rightarrow \operatorname{Re}\left\{\frac{Q_{\beta}^{2-\beta} f(z)}{Q_{\beta}^{3-\beta} f(z)}\right\}>0 \text { in } \mathscr{U} \text {. }
$$

(iii) Let $-1<\beta<2$. If $\frac{Q_{\beta}^{3-\beta} f(z)}{Q_{\beta}^{4-\beta} f(z)}\left(\frac{4}{3}+\frac{Q_{\beta}^{2-\beta} f(z)}{Q_{\beta}^{3-\beta} f(z)}-\frac{4}{3} \frac{Q_{\beta}^{3-\beta} f(z)}{Q_{\beta}^{4-\beta} f(z)}\right)$

$$
\prec \frac{1+\frac{3 B\left[\ln (1-B)+B-\left(B^{2} / 2\right)\right]}{B^{3}+3\left[\ln (1-B)+B-\left(B^{2} / 2\right)\right]} z}{1+B z} \Rightarrow \operatorname{Re}\left\{\frac{Q_{\beta}^{3-\beta} f(z)}{Q_{\beta}^{4-\beta} f(z)}\right\}>0 \text { in } \mathscr{U} \text {. }
$$

Letting $B=-1$ in Corollary 2.12, we have

Corollary 2.13. (i) Let $-1<\beta<0$. If $\operatorname{Re}\left\{\frac{Q_{\beta}^{1-\beta} f(z)}{Q_{\beta}^{2-\beta} f(z)}\left(2+\frac{Q_{\beta}^{-\beta} f(z)}{Q_{\beta}^{1-\beta} f(z)}-2 \frac{Q_{\beta}^{1-\beta} f(z)}{Q_{\beta}^{2-\beta} f(z)}\right)\right\}$

$>\frac{2 \ln 2-1}{2 \ln 2-2} \approx-0.61 \Rightarrow \operatorname{Re}\left\{\frac{Q_{\beta}^{1-\beta} f(z)}{Q_{\beta}^{2-\beta} f(z)}\right\}>0$ in $\mathscr{U}$.

(ii) Let $-1<\beta<1$. If $\operatorname{Re}\left\{\frac{Q_{\beta}^{2-\beta} f(z)}{Q_{\beta}^{3-\beta} f(z)}\left(\frac{3}{2}+\frac{Q_{\beta}^{1-\beta} f(z)}{Q_{\beta}^{2-\beta} f(z)}-\frac{3}{2} \frac{Q_{\beta}^{2-\beta} f(z)}{Q_{\beta}^{3-\beta} f(z)}\right)\right\}$

$>\frac{4 \ln 2-3}{4 \ln 2-2} \approx-0.29 \Rightarrow \operatorname{Re}\left\{\frac{Q_{\beta}^{2-\beta} f(z)}{Q_{\beta}^{3-\beta} f(z)}\right\}>0$ in $\mathcal{U}$.

(iii) Let $-1<\beta<2$. If $\operatorname{Re}\left\{\frac{Q_{\beta}^{3-\beta} f(z)}{Q_{\beta}^{4-\beta} f(z)}\left(\frac{4}{3}+\frac{Q_{\beta}^{2-\beta} f(z)}{Q_{\beta}^{3-\beta} f(z)}-\frac{4}{3} \frac{Q_{\beta}^{3-\beta} f(z)}{Q_{\beta}^{4-\beta} f(z)}\right)\right\}$

$$
>\frac{12 \ln 2-19}{12 \ln 2-20} \approx 0.91 \Rightarrow \operatorname{Re}\left\{\frac{Q_{\beta}^{3-\beta} f(z)}{Q_{\beta}^{4-\beta} f(z)}\right\}>0 \text { in } \mathscr{U} \text {. }
$$


Letting $A=1-2 \delta, 0 \leq \delta<1$ and $B=-1$ in Corollaries 2.9, 2.10 and 2.11, respectively, we have

Corollary 2.14. (i) Let $-1<\beta<0$. If $\operatorname{Re}\left\{\frac{Q_{\beta}^{1-\beta} f(z)}{Q_{\beta}^{2-\beta} f(z)}\left(2+\frac{Q_{\beta}^{-\beta} f(z)}{Q_{\beta}^{1-\beta} f(z)}-2 \frac{Q_{\beta}^{1-\beta} f(z)}{Q_{\beta}^{2-\beta} f(z)}\right)\right\}>\delta$

$\Rightarrow \operatorname{Re}\left\{\frac{Q_{\beta}^{1-\beta} f(z)}{Q_{\beta}^{2-\beta} f(z)}\right\}>(2 \delta-1)+2(1-\delta) \ln 2$.

(ii) Let $-1<\beta<1$. If $\operatorname{Re}\left\{\frac{Q_{\beta}^{2-\beta} f(z)}{Q_{\beta}^{3-\beta} f(z)}\left(\frac{3}{2}+\frac{Q_{\beta}^{1-\beta} f(z)}{Q_{\beta}^{2-\beta} f(z)}-\frac{3}{2} \frac{Q_{\beta}^{2-\beta} f(z)}{Q_{\beta}^{3-\beta} f(z)}\right)\right\}>\delta$

$\Rightarrow \operatorname{Re}\left\{\frac{Q_{\beta}^{2-\beta} f(z)}{Q_{\beta}^{3-\beta} f(z)}\right\}>(2 \delta-1)-4(1-\delta)(\ln 2-1)$.

(iii) Let $-1<\beta<2$. If $\operatorname{Re}\left\{\frac{Q_{\beta}^{3-\beta} f(z)}{Q_{\beta}^{4-\beta} f(z)}\left(\frac{4}{3}+\frac{Q_{\beta}^{2-\beta} f(z)}{Q_{\beta}^{3-\beta} f(z)}-\frac{4}{3} \frac{Q_{\beta}^{3-\beta} f(z)}{Q_{\beta}^{4-\beta} f(z)}\right)\right\}>\delta$

$\Rightarrow \operatorname{Re}\left\{\frac{Q_{\beta}^{3-\beta} f(z)}{Q_{\beta}^{4-\beta} f(z)}\right\}>(2 \delta-1)+3(1-\delta)(2 \ln 2-3)$.

\section{Acknowledgements}

The author would like to thank the referee for his helpful comments and suggestions.

\section{References}

[1] M. Abramowitz and I.A. Stegun, Hand Book of Mathematical functions, Dover Publications, Inc., New York, 1971.

[2] S.D. Bernardi, Convex and starlike univalent functions, Trans. Amer. Math. Soc., 135 (1969), 429-446.

[3] S.D. Bernardi, The univalence of certain analytic functions, Proc. Amer. Math. Soc., 24(1970), 312-318.

[4] I.B. Jung, Y.C. Kim and H.M. Srivastava, The Hardy space of analytic functions associated with certain oneparameter families of integral operators, J. Math. Anal. Appl., 179 (1993), 138-147.

[5] S.S. Miller and P.T. Monacu, Differential subordination and univalent functions, Michigan Math. J., 28 (1981), 157-171.

[6] S. Owa and H. M. Srivastava,Some applications of the generalized Libera operator, Proc. Japan Acad., 62 (1986), 125-128.

[7] H.M. Srivastava and S. Owa, New characterization of certain starlike and convex generalized hypergeometric functions, J. Nat. Acad. Math. India, 3(1985), 198-202.

[8] H.M. Srivastava and S. Owa, A certain one-parameter additive family of operators defined on analytic functions, J. Anal. Appl., 118(1986), 80-87.

Faculty of Science, Department of Mathematics, Al al-Bayt University, P.O. Box: 130095 Mafraq, Jordan.

E-mail: bafrasin@yahoo.com 